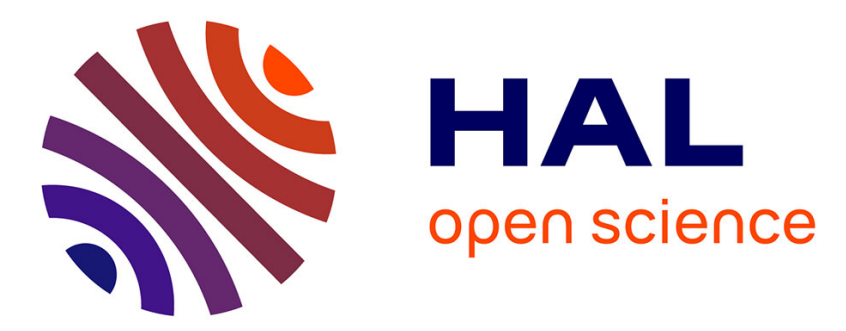

\title{
Women2Win et la féminisation du Parti conservateur : les coulisses d'un débat
}

Véronique Molinari

\section{To cite this version:}

Véronique Molinari. Women2Win et la féminisation du Parti conservateur: les coulisses d'un débat.

Observatoire de la société britannique, 2008, 6, pp.125-143. hal-00911649

\section{HAL Id: hal-00911649 \\ https://hal.science/hal-00911649}

Submitted on 29 Nov 2013

HAL is a multi-disciplinary open access archive for the deposit and dissemination of scientific research documents, whether they are published or not. The documents may come from teaching and research institutions in France or abroad, or from public or private research centers.
L'archive ouverte pluridisciplinaire $\mathbf{H A L}$, est destinée au dépôt et à la diffusion de documents scientifiques de niveau recherche, publiés ou non, émanant des établissements d'enseignement et de recherche français ou étrangers, des laboratoires publics ou privés. 


\title{
Observatoire de la société britannique
}

6 (2008)

Les coulisses du pouvoir

Véronique Molinari

\section{Women2Win et la féminisation du Parti conservateur: les coulisses d'un débat}

\begin{abstract}
Avertissement
Le contenu de ce site relève de la législation française sur la propriété intellectuelle et est la propriété exclusive de l'éditeur.

Les œuvres figurant sur ce site peuvent être consultées et reproduites sur un support papier ou numérique sous réserve qu'elles soient strictement réservées à un usage soit personnel, soit scientifique ou pédagogique excluant toute exploitation commerciale. La reproduction devra obligatoirement mentionner l'éditeur, le nom de la revue, l'auteur et la référence du document.

Toute autre reproduction est interdite sauf accord préalable de l'éditeur, en dehors des cas prévus par la législation en vigueur en France.
\end{abstract}

revues.org

Revues.org est un portail de revues en sciences humaines et sociales développé par le Cléo, Centre pour l'édition électronique ouverte (CNRS, EHESS, UP, UAPV).

Référence électronique

Véronique Molinari, « Women2Win et la féminisation du Parti conservateur : les coulisses d'un débat », Observatoire de la société britannique [En ligne], 6 | 2008, mis en ligne le 01 février 2011, consulté le 14 novembre 2013. URL: http://osb.revues.org/449; DOI : 10.4000/osb.449

Éditeur : Université du Sud-Toulon-Var

http://osb.revues.org

http://www.revues.org

Document accessible en ligne sur :

http://osb.revues.org/449

Document généré automatiquement le 14 novembre 2013. La pagination ne correspond pas à la pagination de l'édition papier.

Tous droits réservés 


\author{
Véronique Molinari
}

\title{
Women2Win et la féminisation du Parti conservateur: les coulisses d'un débat
}

\author{
Pagination de l'édition papier : p. 125-143
}

Les enjeux électoraux représentés par l'électorat féminin n'ont eu de cesse, au cours de ces dix dernières années, d'être évoqués à la veille et au lendemain de chaque élection parlementaire britannique, par la presse, les organisations féministes et les partis eux-mêmes. Les travaillistes les premiers, suite à leur quatrième défaite électorale face aux conservateurs en 1992, ont compris que la reconquête du pouvoir passait par la conquête de l'électorat féminin, jugé comme traditionnellement acquis à leurs adversaires. Celle-ci nécessitait, comme pour le reste de l'électorat, une modernisation du parti, mais également une réorientation du discours vers des préoccupations plus proches des femmes et une féminisation du parti lui-même.

Face à la féminisation progressive de son principal adversaire et l'adoption, par ce dernier, de mesures d'action positive telles que les listes de sélection composées uniquement de femmes (pour les élections législatives à Westminster) ou le jumelage de circonscriptions ${ }^{1}$ (pour les élections au Parlement écossais et à l'Assemblée galloise), le Parti conservateur s'est toujours dit opposé à de telles mesures, jugées anti-démocratiques et « anti-méritocratiques ». Depuis les élections de 2001, toutefois, certaines voix ont commencé à s'élever en son sein et à remettre en question la politique du parti en matière de représentation féminine, mettant en garde les dirigeants contre le fait que l'immobilisme en la matière conduirait tout droit vers de nouvelles défaites électorales ${ }^{2}$. De fait, en mai 2005, en dépit d'un Premier ministre au plus bas dans les sondages, les travaillistes remportaient leur troisième victoire consécutive. L'apparition, quelques mois plus tard de Women 2 Win, groupe de pression conservateur visant à augmenter le nombre d'élues conservatrices et l'intérêt accordé par David Cameron à la question de la représentation féminine au sein du parti, sont à replacer dans ce contexte.

Cet article se propose d'examiner les différentes stratégies adoptées pour promouvoir une plus grande représentation féminine au sein du Parti conservateur ainsi que les résultats obtenus et les résistances rencontrées, notamment au niveau local. Nous verrons ainsi que les initiatives et les débats suscités par la question ont été en grande partie engagés dans les coulisses du parti mais que la place grandissante occupée par les nouvelles technologies de l'information a, dans le même temps, contribué à déplacer cette dernière sur le devant de la scène.

\section{La perte de l'électorat féminin}

Depuis leur accession à l'électorat en 1918 jusqu'aux années 1990, les femmes ont systématiquement été plus nombreuses à voter conservateur que les hommes. Cet écart entre vote féminin et vote masculin, connu en anglais sous le nom de "gender gap" et qui a culminé au début des années 1970, est tel qu'il a été calculé que, dans l'hypothèse où les femmes n'auraient jamais exercé leur droit de vote, les travaillistes seraient restés au pouvoir de façon ininterrompue depuis $1945^{3}$. En 1992, lors de la dernière victoire électorale des conservateurs, elles étaient encore $44 \%$ contre $41 \%$ des hommes à voter pour le parti conservateur. ${ }^{4}$ Depuis, toutefois, le soutien des électrices pour les conservateurs est tombé à $32 \%$ aux élections de mai 1997, $33 \%$ en 2001, $27 \%$ en 2003 et $32 \%$ aux élections de mai 2005 (Tableau 1), chute qui concerne également l'électorat le plus fidèle des conservateurs : les femmes de plus de 55 ans, qui représentent près de 9 millions d'électeurs, parmi les plus enclins à voter (environ 1 vote sur $5^{5}$ ). 
Tableau 1 : pourcentage des voix obtenues par le Parti conservateur de 1974 à 2005 évolution du vote hommes/femmes

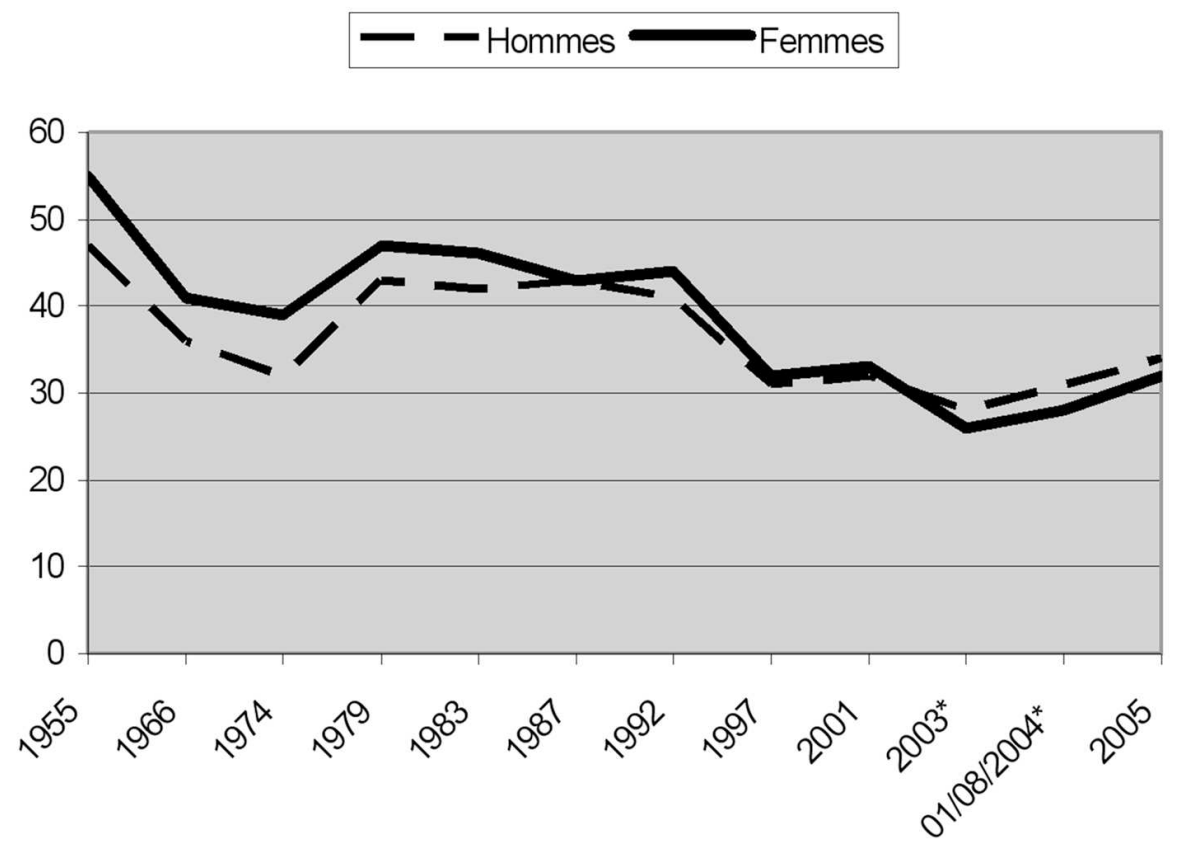

Source : Katherine Rake, "Women voters and the Conservative Party, October 2004 update", Fawcett Society, October 2004, p. 2 ; Gallup, résultats des sondages de 1955 à 1987, dans Pippa NORRIS, "Mobilising the 'Women's Vote" , op. cit. p. 335 ; Sondages Ipsos-MORI/Times, résultats des sondages de 1997, 2001 et 2005.

Les électrices issues de la classe moyenne et des professions libérales, qui étaient encore $43 \%$ à voter conservateur en 1997, ne sont plus que $41 \%$ à le faire en 2001 et plus que $34 \%$ à déclarer soutenir le parti en $2003^{6}$. Les plus grandes pertes concernent les $25-34$ ans, avec huit points de perdus entre 1997 et 2004 (elles ne sont plus que $20 \%$ alors à soutenir le parti) ${ }^{7}$. De fait, les élections suivantes, en mai 2005, se soldent par une troisième défaite consécutive pour les conservateurs : on calcule même qu'avec un électorat exclusivement féminin, les travaillistes auraient obtenu une majorité plus importante ( 90 sièges au lieu des 66 obtenus) et qu'à l'inverse, avec un électorat entièrement masculin, la majorité travailliste aurait été réduite à environ 20 sièges ${ }^{8}$.

Pour certains membres du Parti conservateur, y compris certains ministres et anciens ministres du cabinet fantôme, parmi lesquels Theresa May et Andrew Lansley, la perte de l'électorat féminin et les défaites de 2001 et 2005 sont la conséquence prévisible de l'incapacité du parti à se moderniser et à offrir une image dans laquelle les électeurs et les électrices peuvent se reconnaître ${ }^{9}$. Ils accusent l'absence presque totale, parmi les députés conservateurs, de membres issus des minorités ethniques (ils ne sont que deux députés en 2005) et de femmes (17), qu'ils contrastent avec la situation au sein du Parti travailliste. Ce dernier, dont la victoire de 1997 a largement été attribuée aux électrices, compte alors 27,5\% de députées à Westminster et les libéraux-démocrates $16 \%$, contre $8,6 \%$ seulement pour les conservateurs (voir tableau $\mathrm{n}^{\circ} 2$ ). La question est d'autant plus préoccupante que certains rapports récents montrent qu'une large majorité des électeurs $\left(70 \%{ }^{10}\right)$ souhaitent voir plus de femmes au Parlement et sont plus susceptibles d'aller voter lorsque le candidat est une candidate ${ }^{11}$

Tableau 2 : pourcentage d'élues à la Chambre des Communes dans les trois principaux partis 1983-2005

\begin{tabular}{|l|l|l|l|l|l|l|}
\hline & $\mathbf{1 9 8 3}$ & $\mathbf{1 9 8 7}$ & $\mathbf{1 9 9 2}$ & $\mathbf{1 9 9 7}$ & $\mathbf{2 0 0 1}$ & $\mathbf{2 0 0 5}$ \\
\hline Travaillistes & $4,8 \%$ & $9,2 \%$ & $13,7 \%$ & $24,2 \%$ & $23 \%$ & $27,5 \%$ \\
\hline Conservateurs & $3,3 \%$ & $4,5 \%$ & $6,0 \%$ & $7,8 \%$ & $8,4 \%$ & $8,6 \%$ \\
\hline $\begin{array}{l}\text { Libéraux/Lib- } \\
\text { Démocrates }^{12}\end{array}$ & $0 \%$ & $4,5 \%$ & $10 \%$ & $6,5 \%$ & $9,6 \%$ & $16,1 \%$ \\
\hline
\end{tabular}




\begin{tabular}{|l|l|l|l|l|l|l|}
\hline Autres & $0 \%$ & $8,7 \%$ & $12,5 \%$ & $10 \%$ & $13 \%$ & $10 \%$ \\
\hline Total & $3,5 \%$ & $6,3 \%$ & $9,2 \%$ & $18,2 \%$ & $17 \%$ & $19,8 \%$ \\
\hline
\end{tabular}

\section{La création de Women2Win}

C'est avec pour objectif de remédier à cet état de fait et reconquérir les électrices perdues en 1997 que Women2Win voit le jour en novembre 2005, à quelques semaines de l'élection d'un nouveau chef du Parti conservateur ${ }^{13}$. L'origine directe de l'initiative est à rechercher le mois précédent, à l'occasion du Congrès du parti à Blackpool. Dans les coulisses, une réunion parallèle de la Conservative Women's Organisation, "Women and 21st century politics", organisée le 5 octobre 2005 en marge du Congrès et à laquelle ont participé la Fawcett Society et l'Equal Opportunity Commission, a en effet permis à un certain nombre de militantes de faire part de leur expérience et de dénoncer les difficultés rencontrées en tant que candidates au moment des sélections ${ }^{14}$. La conclusion à laquelle les militantes conservatrices sont parvenues à l'issue de la réunion était la nécessité d'agir rapidement. C'est ainsi que Women2Win est formé, le 23 novembre, par Anne Jenkin, Shireen Ritchie et Theresa May ${ }^{15}$. Très vite, le groupe se dote d'un site web sur lequel il annonce ses objectifs, explique ses actions et encourage les adhésions. Il s'y présente comme « un large échantillon d'hommes et de femmes de tous bords $d u$ Parti conservateur qui reconnaissent que le parti doit changer s'il veut gagner la confiance des Britanniques ${ }^{16}$. Il est nécessaire, pour cela, souligne-t-il, de laisser des candidates disputer des sièges gagnables et d'élire plus de femmes conservatrices au Parlement. Women2Win prône pour cela l'adoption de mesures d'action positive visant à promouvoir la sélection d'un plus grand nombre de candidates aux prochaines élections. Depuis l'élection de Margaret Thatcher à la tête du Parti conservateur puis du pays, trente ans plus tôt, fait remarquer le groupe, le parti n'a pas su évoluer avec la société britannique. Il est désormais urgent pour lui, après une troisième défaite électorale successive, de changer d'image et d'assurer ainsi ses chances d'être ré-élu. Francis Maud, le président du parti, leur accorde immédiatement son soutien.

Les objectifs de Women2Win, sont communiqués aux deux candidats dans la course pour la direction du Parti conservateur : David Davis et David Cameron. Il s'agit de créer une nouvelle culture au sein de ce dernier ${ }^{17}$, nommer un plus grand nombre de femmes à des postes décisionnels au sein du parti, sélectionner plus de candidates dans des circonscriptions acquises et développer un programme qui corresponde aux préoccupations des femmes et à la GrandeBretagne d'aujourd'hui ${ }^{18}$ :

While we talk about defence and deregulation, families up and down the country were looking for us to tell them what we would do to help them care for an elderly relative or a disabled child. Of course our policies on immigration and Europe matter, but to most people, their difficulties with childcare or their child's dependency on drugs matter more. The countries priorities have changed, and so must ours ${ }^{19}$.

L'organisation est opposée aux listes de candidats présélectionnés entièrement composées de femmes (all-women shortlists), telles que celles utilisées par le Parti travailliste pour les élections législatives de 1997 et les dernières élections à l'Assemblée galloise. Elle réclame néanmoins une réforme des procédures de sélection et préconise notamment la mise en place d'une liste prioritaire, composée d'au moins $50 \%$ de femmes et $10 \%$ de minorités ethniques, à partir de laquelle les associations locales de cent « circonscriptions cibles » auraient à choisir leur candidat aux prochaines élections ${ }^{20}$. Aux élections parlementaires de mai 2005, les femmes n'avaient en effet représenté que $12 \%$ des candidats sélectionnés dans les cinquante circonscriptions données comme gagnantes contre $42 \%$ des candidats dans les cinquante circonscriptions les moins susceptibles d'être remportées.

10 Cette proposition d'une liste prioritaire, connue sous le nom de "A-List", est immédiatement adoptée par le nouveau chef du Parti conservateur, David Cameron, élu le 6 décembre 2005 à une large majorité (plus de $67 \%$ des suffrages) ${ }^{21}$. Celui-ci a basé sa campagne sur le thème du changement et de la modernisation et, dès son discours d'investiture, se réfère à la nécessité de remédier à la sous-représentation des femmes au sein du parti ${ }^{22}$ : 
I said when I launched my campaign that we needed to change in order to win. Now that I have won we will change.

We will change the way we look. Nine out of ten Conservative MPs, like me, are white men. We need to change the scandalous under-representation of women in the Conservative party and we will do that. We need to change the way we feel. No more grumbling about modern Britain. I love this country as it is, not as it was. And I believe our best days lie ahead ${ }^{23}$.

La semaine suivante, à Leeds, il annonce la suspension des sélections et l'élaboration de la A-List à partir de laquelle tous les sièges cibles (target seats) ainsi que les circonscriptions actuellement détenues par les conservateurs devront sélectionner leurs candidats. C'est Shireen Ritchie, l'une des fondatrices de Women2Win, qui est d'ailleurs choisie en janvier 2006 pour présider la Commission des candidatures (Candidates' Committee). Deux autres membres du groupe - Theresa May et Bernard Jenkin- sont, avec Fiona Hodgeson, présidente de la $C W O$, chargés de superviser le déroulement des sélections et de mettre en place un programme intensif de recrutement de nouveaux visages ainsi que de mentoring afin d'aider les candidates potentielles dans leurs efforts. La décision est accueillie, par Women2Win, comme "An Historic Step to Winning the Women's Vote" :

In 1997 we lost the women's vote, and we have so far failed to win it back. This decision will send a signal to women across the country that we are a party that not only wants their vote, but also wants their expertise. We must now start a campaign to win the trust and confidence of Britain's women $^{24}$.

Dans les mois qui suivent, diverses stratégies se mettent en place en coulisses. Différentes rencontres sont organisées entre l'équipe de David Cameron et la Fawcett Society, qui fait campagne pour l'égalité homme-femmes, afin d'en apprendre plus sur les attentes des femmes. Women 2 Win, de son côté, organise une série de réunions à Manchester, Londres, Birmingham, Leeds..., des séminaires d'introduction à la politique, des ateliers de prise de parole en public, et fournit conseils, aide pratique et soutien financier aux femmes qui souhaiteraient se porter candidates pour le parti.Un Conservative Women's Forum est également mis en place par la $C W O$ afin d'organiser des réunions-débats sur les projets et propositions de lois actuellement débattus au Parlement. L'objectif est de donner aux femmes, qu'elles appartiennent ou non au Parti conservateur, la possibilité d'avoir un contact direct informel, hors du cadre parlementaire, avec les députés et ministres du cabinet fantôme conservateurs, de poser des questions et de faire part de leur point de vue. Celles qui ne peuvent pas assister à ces réunions (tenues à Millbank House, à proximité du Parlement, à Londres) peuvent faire connaître leur opinion via internet, grâce au Forum de la $C W O$.

\section{La mise en place de la A-List et les réactions}

La A-List est finalement mise en place début 2006 et, au mois de mai, une première liste de 140 candidats, dont plus de $50 \%$ de femmes, est révélée à la presse. Le profil des candidats est, à première vue, assez varié (même si leur expérience professionnelle est, somme toute, assez similaire à celle des précédents députés conservateur ${ }^{25}$ ) et inclut un certain nombre de célébrités, ce qui lui vaut une publicité importante de la part de la presse. Certains, parmi les travaillistes, dénoncent aussitôt une simple opération de marketing. Pour David Cameron, il s'agit avant tout de montrer à la population que son parti a changé : le dirigeant conservateur n'hésite d'ailleurs pas à comparer l'adoption d'une liste prioritaire à l'abandon de la Clause 4 par le parti travailliste en $1995^{26}$. Le mois suivant, en juin 2006, les efforts du Parti conservateur se poursuivent d'ailleurs avec la mise en place du Conservative Muslim Women's Group au sein du CWO « afin de promouvoir une plus grande compréhension et un meilleur dialogue avec les Musulmans vivant au Royaume-Uni $»^{27}$.

14 La féminisation du parti, selon Shireen Ritchie et les autres membres de Women2Win, n'implique toutefois pas seulement d'augmenter le nombre de femmes au sein des députés et de varier le profil de ces derniers mais également de redéfinir la politique du Parti conservateur afin de la rendre plus proche des préoccupations identifiées comme féminines ${ }^{28}$ telles que le système de santé et l'éducation. Ce changement a déjà été amorcé par David Cameron qui, comme Tony Blair dix ans auparavant, n'hésite pas à jouer de son image de jeune père : sur 
son blog, lancé fin septembre 2006, il se présente dans sa cuisine, en train de nettoyer les bols qui ont servi au petit-déjeuner de ses enfants pendant que son bébé pleure dans sa chaise haute et que du linge sèche sur l'étendage ${ }^{29}$. Une semaine plus tard, lors du congrès du parti à Bournemouth, il s'adresse directement aux électrices dans un discours très éloigné du discours conservateur traditionnel dans lequel il mentionne les violences domestiques, les horaires de travail aménagés et l'aide aux mères célibataires en matière de garde d'enfants.

Le processus de modernisation et de féminisation du parti encouragé par Women2Win et soutenu par David Cameron n'est pas sans rencontrer une forte résistance au sein du parti. Les procédures de sélection qui s'opèrent au niveau local, notamment, sont très tôt attaquées par les associations de circonscription, jalouses de leur autonomie. Ainsi, lorsque, fin juillet, une élection partielle est organisée à Bromley et Chislehurst (Grand Londres), suite à la mort du député conservateur Eric Forth ${ }^{30}$ au mois de mai, les militants locaux annoncent immédiatement qu'ils ne tiendront pas compte de la A-List. Ils préfèrent sélectionner Bob Neill, avocat blanc de 53 ans, plutôt qu'une femme (Julia Manning) ou un candidat asiatique (Syed Kamall, député européen) de la liste. "Je n'accepte pas que des minorités ethniques et des femmes soient proposés dans l'unique but d'augmenter leur nombre », déclare à cette occasion Dennis Chantry, vice-président du Club conservateur de Bromley et Chislehurst. Le Daily Telegraph cite un autre responsable de la Conservative Association de Bromley and Chislehurst : "Nous somme une association autonome. Selon la constitution du Parti conservateur, il n'existe aucune A-List ${ }^{31}$. Bob Neill remporte l'élection partielle, mais le Parti conservateur voit sa majorité chuter de 13000 à 633 en faveur des libéraux-démocrates. $\mathrm{Si}$ les tensions entre les associations locales et le Parti conservateur national se déroulent en grande partie en coulisses, des attaques contre la A-List sont également lancées par la « droite Tory », notamment le Tory Cornerstone Group ${ }^{32}$, créé en juillet 2005, qui utilise quant à lui son site web ainsi que les blogs de ConservativeHome pour dénoncer les choix stratégiques et le repositionnement politique du parti vers le centre. Son président, John Hayes, ministre du Cabinet fantôme, encourage ainsi les partis locaux à « résister au politiquement correct » et déclare, en mai 2006 :

The idea that we can parachute insubstantial and untested candidates with little knowledge of the local scene into key seats to win the confidence of people they seek to represent is the bizarre theory of people who spend too much time with the pseuds and posers of London's chichi set and not enough time in normal Britain ${ }^{33}$.

Pour Anne Widdecombe, députée et ancienne ministre conservatrice associée au groupe, la A-List est une insulte envers les femmes et crée deux catégories de députés: " those who have beaten off all competition to get there, and women who have had their paths artificially smoothed". "Neither Margaret Thatcher nor I needed this kind of help to get into Parliament", déclare-t-elle au Daily Mail ${ }^{34}$.

Le premier bilan de la A-List, dressé début juillet 2006, révèle quoi qu'il en soit des résultats mitigés. En effet, si la proportion de femmes sélectionnées au total est tout à fait encourageante (14 sur 46, soit 30,4\%), le pourcentage de candidates sélectionnées depuis la mise en place de la liste prioritaire (29.2\%) est en réalité inférieur à celui des candidates sélectionnées avant $(31.8 \%)^{35}$. Au total, début août, moins d'un tiers des sièges a été pourvu par des candidates, soit la même proportion que sous Michael Howard. Dans $90 \%$ des cas, les associations qui ont rejeté la A-List l'ont fait pour sélectionner un homme ${ }^{36}$.

Le parti s'était jusqu'alors refusé à forcer les associations de circonscription à utiliser la liste. Dans les mois qui suivent, cependant, David Cameron, tente de se montrer plus ferme. Il décide d'augmenter la taille de la A-List ainsi que la proportion de femmes sur celle-ci (60\%) et de changer les procédures de sélection des candidats parlementaires : les associations de circonscriptions cibles devront choisir au moins deux femmes sur une liste de quatre candidats (pratique fort semblable aux quotas, auxquels le parti est longtemps resté opposé) et la sélection finale, après des entretiens approfondis, reviendra au comité exécutif de la circonscription avant approbation par les militants. La mesure est, dit-il, nécessaire s'il veut tenir sa promesse 
d'augmenter le nombre d'élues au sein du Parti conservateur ${ }^{37}$. Accusé de priver les militants de leur droits, Cameron utilise le site de ConservativeHome pour répondre aux attaques :

[...] Commentators [...] have made much of the reforms being anti-meritocratic. But does anyone actually believe that meritocracy has applied in the past? It cannot be that the Parliamentary party is 91 per cent male and 99 per cent white, because the men who apply to be candidates are 91 per cent better than the women, and whites are 99 per cent better than minority ethnic candidates. The sad fact is that we have not been open to all the talents of the country in the way that a properly meritocratic party should be $\mathrm{e}^{38}$.

Les critiques continuent néanmoins de pleuvoir et, fin novembre, alors que la proportion de femmes sélectionnées a augmenté, trois militants tentent - en vain - de mettre fin à la $A$ List en proposant une motion à cet effet au Conseil de Direction du Parti conservateur. John Stafford, l'un d'eux et président de la Campaign for Conservative Democracy ${ }^{39}$, dénonce le fait qu'il est beaucoup plus difficile pour un homme que pour une femme de se faire inscrire sur la liste ${ }^{40}$ et accuse les membres du Central Office responsables de la mise en place de la liste de vouloir tout contrôler ${ }^{41}$. Le 29 janvier 2007, le Conseil de Direction prend pourtant une autre décision : les associations de circonscription seront désormais libres de sélectionner leurs candidats parmi les membres de la A-List (alors composée de 160 noms) ou sur une liste plus importante, de quelque 800 noms, en échange de la promesse qu'à chaque étape de la sélection, les femmes représenteront au moins $50 \%$ des candidats retenus. La décision est relativement bien acceptée par Women 2 Win et la CWO comte tenu du fait que $38 \%$ de candidates (40 sur 108) ont à ce stade été sélectionnées et que les changements engagés semblent déjà porter leurs fruits.

\section{Quels résultats ?}

Fin 2006, les sondages révèlent en effet un pourcentage grandissant d'électrices favorables à David Cameron. Selon YouGov, enaoût 2006, 43 \% de femmes auraient l'intention de voter conservateur contre $28 \%$ travailliste ${ }^{42}$. Un autre sondage (Populus) publié dans The Times, début novembre 2006, parle de la réapparition du gender gap, citant $37 \%$ de femmes qui déclarent soutenir les conservateurs contre $34 \%$ des hommes ${ }^{43}$. La presse attribue aux femmes l'avance de David Cameron sur les travaillistes et Women2Win voit là la preuve de l'efficacité de la nouvelle stratégie. Si l'on en croit toutefois les chiffres de sondages antérieurs réalisé par YouGov et illustrés par les courbes ci-dessous, l'inversion du vote masculin et du vote féminin est antérieure à la création de Women2Win et à l'arrivée de David Cameron à la tête du parti. Elle correspond toutefois à l'émergence de ce dernier sur la scène politique et au début du processus de modernisation du Parti conservateur ainsi qu'à une période de désaffection des électrices pour le Parti travailliste ${ }^{44}$. L'écart de votes devient, qui plus est, particulièrement flagrant, suite à l'élection du nouveau leader conservateur. 
Tableau 3 : avance des conservateurs dans les sondages YouGov 2004-2006

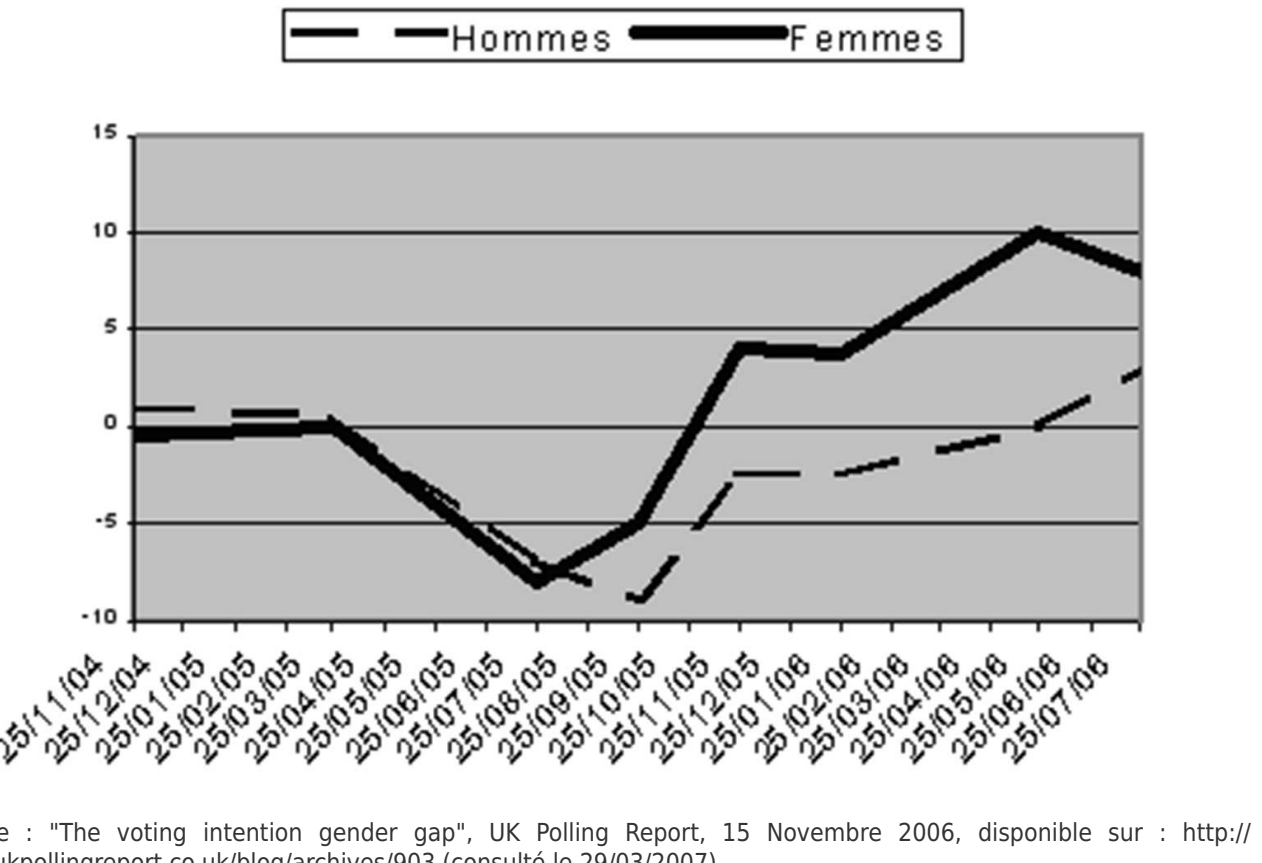

Source : "The voting intention gender gap", UK Polling Report,
www.ukpollingreport.co.uk/blog/archives/903 (consulté le 29/03/2007)

\section{Aspirations féministes ou simple stratégie électorale ?}

La publicité faite par la presse à la $A$-List font dire à certains que les réformes engagées par les conservateurs ne sont rien de plus qu'une opération de publicité destinée à couvrir le fait que le parti ne propose rien de concret en matière de politique en faveur des femmes ${ }^{45}$. Cependant, ni Women2Win ni David Cameron ne cherchent à dissimuler leurs objectifs électoraux : la féminisation du parti fait clairement partie d'une stratégie plus large visant à donner une image plus moderne du Parti conservateur et augmenter ainsi ses chances d'élection. Theresa May déclare ainsi, à propos de la création de Women2Win et de la nécessité d'augmenter la présence féminine dans les rangs conservateurs: "This isn't just about we have women sitting on the benches in the House of Common [...], this is also about how we can win back the women's vote which has won us every election that we've won in the $20^{\text {th }}$ century" ${ }^{\text {"46 }}$. David Cameron, au moment où il est confronté à certaines rébellions de la part des partis locaux en août 2006, reprend les mêmes arguments pour répondre aux attaques dont il est victime :

The aim is clear: we must make our benches in the Commons more truly representative of the country we aspire to govern. It is about fairness, it is about better representation for women - but above all it is about effectiveness. We will only be a really effective political fighting force when we are using the talents of all our people ${ }^{47}$.

Ces déclarations ne signifient pas pour autant que la féminisation du Parti conservateur est purement imputable à des considérations d'ordre stratégique ainsi que certains le laissent entendre. Le féminisme de Theresa May et d'autres militantes conservatrices membres de Women 2 Win ne peut pas être mis en doute. Leurs efforts pour convaincre les instances dirigeantes du parti de promouvoir les candidatures féminines et leurs prises de position en faveur de mesures d'action positive sont en effet bien antérieurs à la création du groupe. En 2002, Theresa May, alors à la présidence du Parti conservateur, défendait déjà l'idée d'une liste de candidats composée à $50 \%$ de femmes ${ }^{48}$. C'est parce que ces demandes avaient toutefois, jusqu'à présent, reçu peu d'attention de la part des organes dirigeants que l'argument de l'efficacité électorale, rendu pertinent par la situation de crise dans laquelle se trouvait leur parti après une troisième défaite électorale, a été utilisé.

\section{Conclusion}

Ce sont les frustrations ainsi que les difficultés des militantes conservatrices à se faire entendre qui ont conduit à la création, en novembre 2005, de Women 2 Win. De la même manière que 
les discussions qui ont précédé, les modalités de mise en place d'une liste prioritaire destinée à redresser le déséquilibre hommes-femmes sur les listes électorales du Parti conservateur ainsi que les débats auxquels cette initiative a donné lieu se sont en grande partie poursuivis en coulisses. Certes l'utilisation des nouvelles technologies de l'information - les sites web en particulier - par le groupe ainsi que par les autres groupes de pression conservateurs a en partie contribué à déplacer le lieu du débat. Dans le cas de Women2Win, toutefois, si l'organisation reste très active en coulisses, le site web du groupe est quant à lui relativement statique et très peu critique de la politique du parti. En dépit des progrès réalisés - les femmes représentent aujourd'hui 22,5 \% des ministres du Cabinet fantôme conservateur contre 22,7 \% du nouveau Cabinet travailliste de Gordon Brown -, les conséquences de la décision du Conseil de direction, en janvier 2007, de laisser les associations de circonscription libres d'utiliser ou non la $A$-List, n'ont pourtant pas tardé à se faire sentir : le pourcentage de candidates sélectionnées a en effet chuté de façon significative, de $38 \%$ en janvier à $33 \%$ aujourd'hui. Fiona Hodgeson, présidente de la Conservative Women's Organisations et l'un des membres chargés de superviser les sélections, assurait en avril 2007 que la Commission des candidatures du parti ne laisserait pas la situation se détériorer ${ }^{49}$ et le Parti Central a, de fait, envisagé, dans les mois qui ont suivi, de rétablir la liste. Aucune initiative n'a, toutefois, été prise à l'heure où nous écrivons, et Women2Win, tout comme la $C W O$, fait preuve du silence le plus total sur la question. Après plusieurs mois passés sur le devant de la scène, le débat se déroule de nouveau là où il avait commencé : dans les coulisses du parti.

\section{Bibliographie}

Mes remerciements à Fiona Hodgson pour sa disponibilité.

Campbell, B., The Iron Ladies. Why Do Women Vote Tories ?, Londres : Virago, 1987.

Campbell, R., Lovenduski, J. \& Norris, P., Gender and Political Participation, Research Report, Londres : Electoral Commission, April 2004.

Garnett, M. \& Lynch, P., The Conservatives in Crisis. The Tories After 1997, Manchester : Manchester University Press, 2003.

Gyimah, S., From the Ashes : the Future of the Conservative Party : Leading Politicians and Commentators Speak Out, Londres : Bow Publications, 2005.

Maguire, G. E., Conservative Women : A History of Women and the Conservative Party, 1874-1997, Londres : Macmillan, 1998.

Norris, P., "Mobilizing the 'Women's Vote' : The Gender-Generation Gap in Voting Behaviour" Parliamentary Affairs 49.2, 1996, pp. 333-42.

Norris, P., "The Gender Gap in Britain and America." Parliamentary Affairs 38.2, 1985, pp. 192-201.

Rake, K., "Women voters and the Conservative Party, October 2004 update", Fawcett Society, October 2004. http://www.fawcettsociety.org.uk/documents/Women_and_Conservatives_Oct04.pdf, consulté le 4-12-2004.

Turquet, L., "Women's Votes and the Conservative Party 2003", Fawcett Society, Based on resarch by MORI with aditional material by Roger Mortimore, Rachel Williams and Emma Wainwright, p. 2.

\section{Sites internet :}

Fawcett Society : http://www.fawcettsociety.org.uk

Conservative Party : http://conservatives.com

Ipsos-Mori : http://www.ipsos-mori.com

Women2Win : http://www.women2win.com

\section{Notes}

1 Le procédé, qui a uniquement été utilisé pour les élections de 1999, consistait à « jumeler » des circonscriptions géographiquement et politiquement proches afin de former un comité de sélection 
unique qui aurait à sélectionner deux candidats, un homme et une femme, chaque candidat se présentant ensuite pour un siège.

2 "Twice we went to the country, unchanged, unrepentant, just plain unattractive, and twice we were slaughtered", déclare Theresa May, alors Présidente du parti, dans son discours au congrès du Parti conservateur de Bournemouth en septembre 2002. Mark Davies, "Tories must change or face slaughter", BBC News, 7/09/2002.

3 Voir Pippa Norris, "The Gender Gap in Britain and America." Parliamentary Affairs 38.2, 1985, pp. 192-201 et "Mobilizing the 'Women's Vote' : The Gender-Generation Gap in Voting Behaviour" Parliamentary Affairs 49.2, 1996, pp. 333-342. Les différences de comportements électoraux ne sont toutefois pas uniquement liées au sexe de l'électeur mais également à son âge. Ainsi, les femmes âgées ont tendance à voter à droite en plus grand nombre que les hommes du même âge, tandis que les jeunes femmes votent plus à gauche que les électeurs du même âge. Pippa Norris remarque à ce sujet qu'il est plus juste de parler de "gender-generation gap" (Pippa NORRIS, "Mobilizing the 'Women's Vote' : The Gender-Generation Gap in Voting Behaviour", op. cit., p. 333).

4 Katherine Rake, "Women voters and the Conservative Party, October 2004 update", Fawcett Society, October 2004, p. 2, disponible sur : http://www.fawcettsociety.org.uk/documents/ Women_and_Conservatives_Oct04.pdf (consulté le 4/12/2004).

$569 \%$ de votant parmi les 55-64 ans et $70 \%$ parmi les 65 ans et plus aux élections de mai 2001 pour un taux de participation global de $59 \%$; $71 \%$ de votants parmi les 55-64 ans et $75 \%$ parmi les 65 ans et plus aux élections de mai 2005 pour un taux de participation global de $61 \%$ (source : IPSOS-MORI , "How Britain Voted in 2001", http://www.ipsos-mori.com/polls/2001/election.shtml; IPSOS-MORI, "Who Voted Which Way - In Detail", http://www.ipsos-mori.com/polls/2005/electionaggregate.shtml (consulté le 5/03/2007)

6 Laura TURQUET, "Women's Votes and the Conservative Party 2003", Fawcett Society, p. 2.

7 Katherine RAKE, "Women voters and the Conservative Party, October 2004 update", op. cit.

8 Parmi celles qui ont voté, $38 \%$ auraient voté travailliste (contre $34 \%$ des hommes), $32 \%$ conservateur (contre $34 \%$ des hommes) et $22 \%$ libéral-démocrate (source : Ipsos-MORI, "Women's Support Gave Blair the Edge", 8 Mai 2005. Echantillon : 18000 personnes), http://www.ipsos-mori.com/publications/ rmw/womens-support-gave-blair-the-edge.shtml (consulté le 2 janvier 2007).

9 "The poor representation of women and the lack of ethnic diversity in the Conservative Party set it apart from the Britain of 2005. We believe that it is because we do not look, talk or sound like modern Britain, that modern Britain has chosen to reject us in the past", "About women2win", http:// www.women2win.com/about.php (consulté le 5/01/2006). C'est également ce qu'explique Theresa May dans Woman's Hour sur BBC Radio Four à l'occasion du lancement de Women2Win, 24 novembre 2005, http://www.bbc.co.uk/radio4/womanshour/2005_47_thu_04.shtm (consulté le 25 janvier 2007).

10 Un chiffre qui atteint les 83 \% pour la tranche d'âge des 16-34 ans. "Fawcett welcomes Conservative candidate reform", 12/12/2005, source : http://www.fawcettsociety.org.uk//index.asp ?pageID =157 (consulté le 28/10/2006).

11 En 2001, le taux de participation électorale était en effet de $4 \%$ plus élevé parmi les femmes que parmi les hommes dans les circonscriptions où une candidate a été élue (le taux de participation des hommes, quant à lui, était le même), Rosie Campbell, Joni Lovenduski et Pippa Norris, Gender and Political Participation, Research Report, avril 2004, p. 9.

12 Libéraux Démocrates à partir de 1992.

13 Michael Howard avait annoncé sa démission en mai 2005, suite à la défaite du parti aux élections parlementaires.

14 Source : Fiona Hodgeson, présidente de la CWO courrier du 6 février 2007.

15 Le groupe est présidé par Theresa May et Brooks Newmark (tous deux députés). Siègent au conseil consultatif (Advisory Board) Sandra Burling, Fiona Hodgeson, Eleanor Laing (députée), Maria Miller (députée), Trish Morris, Shireen Ritchie, Caroline Spelman (députée), Peter Viggers (député).

16 "About women2win", ", http://www.women2win.com/about.php (consulté le 5/01/2006).

17 L'organisation déclare également vouloir adopter un « ton plus positif et plus constructif dans l'Opposition et un style de politique moins agressif ». Women2Win, About us , http:// www.women2win.com/about.php (consulté le 17/02/2007).

18 Women2Win, "Our agenda for Change", http://www.women2win.com/action.php (consulté le 5/01/2006).

19 "Our Agenda for Change", http://www.women2win.com/action.php (consulté le 5/01/2006). De fait, tandis que les électrices classent, parmi leurs priorités, les services publics, et en particulier la santé et l'éducation, l'immigration, qui apparaît en deuxième place pour les hommes n'est que la quatrième priorité pour les femmes. 
20 Selon la vice-présidente du parti, Margot James, l'objectif est d'offrir une image qui reflète celle de la société et de montrer aux femmes, et en particulier aux femmes noires et asiatiques, qu'elles sont maintenant les bienvenues au sein du Parti conservateur. Emily Wilson, "Cameron unveils his new women - and not a blue rinse in sight", The Guardian, 13/05/2006.

21 Si le groupe dit, à sa création, ne soutenir aucun candidat particulier à l'élection à la tête du Parti conservateur, Theresa May a néanmoins déclaré ouvertement son soutien à David Cameron quelques semaines avant l'élection.

22 À noter qu'il fait déjà référence, dans son discours au congrès de Blackpool, en octobre 2005, au fait que le parti conservateur a perdu le soutien des électrices. http://politics.guardian.co.uk/ conservatives2005/ story/0,1584777,00.html (consulté le 11/02/2007).

23 "In full : Cameron victory speech", BBC News, 6 décembre 2005, disponible sur : http:// news.bbc.co.uk/2/hi/uk_news/politics/4504722.stm (consulté le 15/12/2005).

24 "An Historic Step to Winning the Women's Vote Says Campaign Group", 11/12/2005, http:// www.women2win.com/newsstory.php ?newsid =8 (consulté le 7/09/2006)

25 La majorité des candidates est en effet issue du milieu des affaires, vient du Sud de l'Angleterre (61\%) et a déjà une expérience en politiques : les deux tiers, environ, ont déjà disputé des élections quoi que dans des sièges perdus d'avance - et un quart environ sont ou ont été conseillères municipales. Néanmoins, un grand nombre de femmes de la liste sont des mères de famille avec de jeunes enfants, ce qui représente une nouveauté indéniable.

26 "I have never had a clause 4 ; I have never sought to invent one. But the scale of change is as profound". Tania Branigan, "Cameron to increase number of ethnic minority candidates", The Guardian, 26/04/2006.

27 Conservative Women's Muslim Group, http://www.conservativewomen.org.uk/ muslimwomengroup.asp (consulté le 2/05/2007).

28 "Feminising the Conservatives", Woman's Hour, BBC Radio Four, 11 octobre 2006, http://www.bbc.co.uk/radio4/womanshour/02/2006_41_wed.shtml (consulté le 17/02/2007). Selon un sondage réalisé à la veille des élections de mai 2005, le système de santé apparaît en tête des préoccupations des électrices ( $36 \%$ ), suivi de l'éducation (34\%), des impôts, de l'Europe, du terrorisme, de l'Irak, de l'immigration et des congés de maternité et parentaux (11\%). "The Eve/newstateswoman Survey", Newstateswoman, 04/04/2005, p. 18.

29 "Introduction to Webcameron", 29 septembre 2006, http://www.webcameron.org. uk (consulté le 17/02/2007).

30 Lui-même était fortement opposé à l'utilisation de mesures d'action positive. Il avait ainsi déclaré, lors d'une émission de radio au sujet de Women2Win: "This idea that we have to force-feed Parliament with women sounds absurd". BBC Radio Four, Woman's Hour, Women2Win, 24/11/2005, http:// www.bbc.co.uk/radio4/ womanshour/2005_47_thu_04.shtml (consulté le 25/01/2007).

31 Melissa Kite and Jonathan Wynne-Jones, "Grass-root Tories warn of revolt over A-List", Daily Telegraph, 21/05/2006.

32 Le Cornerstone Group, également connu sous le nom de "Faith, Flag and Family", d'après les trois institutions qu'il défend, est soutenu par une quarantaine de députés conservateurs.

33 Ben Hall, "Cameron accused of 'chichi' approach", Financial Times, 30/05/2006.

34 Simon Walters, "Cameron to force Tory 'stick in the muds' to select more women MPs", Daily Mail, 19/08/2006.

35 On compte ainsi 7 femmes sur les 22 candidats sélectionnés avant la mise en place de la A-List et 7 femmes sur les 24 candidats sélectionnés depuis. La proportion de minorités ethniques est quant à elle de 2 sur 46 (soit $4.3 \%$ ).

36 Des différences régionales sont apparues : tandis que Londres, les Midlands et le Nord-Ouest ont sélectionné presque autant de femmes que d'hommes, le Sud-Ouest, l'Est et le Yorkshire (2 femmes seulement) affichent les plus mauvais résultats.

37 Will Woodward, "Cameron tries again to get more women selected", The Guardian 21/08/2006 ; Greg Hurst, "Campaign for more women MPs boosted", The Times, 21/08/2006.

38 "David Cameron responds to your concerns... " 21/08/2006, http://conservativehome.blogs.com/ goldlist/2006/08/david_cameron_r.html (consulté le 10/09/2006).

39 Campaign for Conservative Democracyest un groupe de pression interne au Parti conservateur ayant pour objectif une réforme du parti pour un fonctionnement plus démocratique ainsi que la défense de la démocratie, menacée, selon lui, par les réformes constitutionnelles engagées par le gouvernement travailliste en place. Ilse définit comme "a group of members of the Conservative Party who believe that our democracy is under threat and we must therefore both defend and promote it, including within the Conservative Party", http://www.btinternet.com/ johnstrafford/ (consulté le 15/07/2007). 
40 "It is four and a half times more difficult for men than for women to get on the list, which means that the men who do get on are better than the women". Andy McSmith, "Cameron hails rise in women candidates", The Independent, 23/11/2006.

41 Campaign for Conservative Democracy, http://www.btinternet. com/ johnstrafford/ bulletin.htm\#March \%2018th (consulté le 15/07/2007).

42 Lesley White, "Blue Swoon", The Sunday Times Magazine, 30/08/2006.

43 La différence entre vote féminin et vote masculin est encore plus importante lorsque l'on demande aux électeurs pour qui ils voteraient dans quatre ans si le Parti travailliste était dirigé par Gordon Brown et le Parti conservateur par David Cameron : $42 \%$ des femmes déclarent préférer voter pour les conservateurs (30\% pour les travaillistes) contre $34 \%$ des hommes. Peter Riddell \& Philip Webster, "Women voters are the key to Cameron's lead over Labour", The Time, 7/11/2006.

44 À en croire les différents sondages, la guerre en Irak et les raisons invoquées pour y engager la Grande-Bretagne aux côtés des États-Unis auraient largement contribué à cette perte de confiance. Au total, $63 \%$ de femmes se déclarent, en 2005, mécontentes du gouvernement.

45 Mary Riddell, "Dave's wining way with women voters", The Observer, 28 May 2006 ; Emily Wilson, "Cameron unveils his new women — and not a blue rinse insight", The Guardian, 13 May 2006

46 "Women2Win", Woman's Hour, BBC Radio Four, avec Eric Forth MP and Theresa May MP, 24/11/2005.

47 "David Cameron responds to your concerns", 21/08/2006, http://conservativehome.blogs.com/ goldlist/ 2006/08/david_cameron_r.html (consulté le 10/09/2006).

48 Brian Wheeler, "Quotas an option for Tories - May", BBC News, 5/10/2002, disponible sur http:// news.bbc.co.uk/2/hi/uk_news/politics/2300155.stm (consulté le 10/05/2007). Il est également à noter qu'en octobre 2001, lors des débats sur le Sex Disqualification (Election Candidates) Bill, présenté par le gouvernement travailliste, Theresa May avait soutenu le projet de loi. Celui-ci, visant à autoriser les mesures d'action positive telles que les listes de candidates, le jumelage de circonscriptions, l'alternance homme/femme sur les listes ou encore les quotas lors des élections à la Chambre des Communes, au Parlement écossais, à l'Assemblée nationale galloise, à l'Assemblée nord-irlandaise, au Parlement européen et aux élections locales, a été voté en 2002.

49 Courrier du 23 avril.

\section{Pour citer cet article}

\section{Référence électronique}

Véronique Molinari, «Women2Win et la féminisation du Parti conservateur : les coulisses d'un débat », Observatoire de la société britannique [En ligne], 6 I 2008, mis en ligne le 01 février 2011, consulté le 14 novembre 2013. URL : http://osb.revues.org/449 ; DOI : 10.4000/osb.449

\section{Référence papier}

Véronique Molinari, «Women2Win et la féminisation du Parti conservateur : les coulisses d'un débat », Observatoire de la société britannique, 6 | 2008, 125-143.

\section{À propos de l'auteur}

\section{Véronique Molinari}

Maître de Conférences à l'Université de Grenoble III

\section{Droits d'auteur}

Tous droits réservés

\section{Résumé}

Le 5 mai 2005, le Parti conservateur subissait une troisième défaite consécutive face aux travaillistes, remportant seulement 32,3\% des voix et 198 sièges. Pour certains de ses militants, 
ce résultat était en grande partie la conséquence de l'incapacité du parti à se moderniser et à offrir une image représentative de la société actuelle. L'absence quasi-totale, parmi les députés conservateurs, de minorités ethniques et de femmes, notamment, fut comparée avec les progrès accomplis durant les dix années précédentes par le Parti travailliste dont le succès, depuis 1997, avait largement été attribué à l'électorat féminin. C'est avec pour objectif de reconquérir les électrices perdues en 1997 —et du même coup tenter de reconquérir le pouvoir- que certaines figures du Parti conservateur ont, fin novembre 2005, formé Women2Win, groupe de pression destiné à promouvoir la sélection d'un plus grand nombre de candidates. Cet article se propose d'examiner les stratégies utilisées par ce dernier pour féminiser le partiainsi que les résultats obtenus et les résistances rencontrées. Nous verrons ainsi que les initiatives et les débats suscités par la question ont été en grande partie engagés dans les coulisses du parti, même si la place grandissante occupée par les nouvelles technologies de l'information a partiellement contribué à déplacer cette dernière sur le devant de la scène.

\section{Entrées d'index}

Mots-clés : femmes, parti conservateur, Women2Win 